\title{
On the behavior of rapid thermal CVD reactors
}

\author{
S. AIT AMER, S. MAGNAUDEIX and P. DUVERNEUIL
}

Laboratoire de Génie Chimique, URA 192 du CNRS, ENSIGC/INPT, 18 chemin de la Loge, 31078 Toulouse cedex, France

\begin{abstract}
Two mathematical models are developed and applied to RTCVD reactors. They involve the resolution of momentum, heat and mass transfer equations with homogeneous and heterogeneous chemical reactions. A detailed discussion about the effects of some physical parameters is undertaken so that a fundamental understanding of gas flow patterns and the contribution of each chemical species to the deposition of polysilicon is obtained.
\end{abstract}

\section{1.- Introduction.}

During the last three decades, the evolution of microelectronics has followed two main directions : a reduction of components size and more recently an increase of productivity obtained by increasing the wafers size over $6^{\prime \prime}$. Therefore a new kind of process has been developed : Rapid Thermal Processing (RTP). Initially used for implantation annealing, it has seen its application extented to the elaboration of CVD layers because the classical CVD processes which use hot wall tubular reactors cannot treat large wafers [1]. The equipments used in RTP are typically cold wall reactors where the external walls are cooled and only the substrate is heated by radiation. The main advantage of this type of reactors consists in the rapid increase and decrease of temperature, which enables to accelerate the treatment.

In this paper, a modeling work concerning RTCVD reactors will be presented. Two different models will be used to determine the velocity, temperature and concentration fields. The first one has been elaborated especially to simulate cold wall axi-symmetrical reactors and the second one, ESTET, is commercially available.

The influences of operating conditions and geometrical characteristics will be pointed out in order to help future reactor designs and the determination of optimal functioning conditions for depositions by RTCVD. As an example, the deposition of pure polysilicon will be studied to illustrate our purpose.

\section{2.- Reactor geometry.}

A schematic cross-sectional view of the reactor which has been studied is shown in Fig.1. The apparatus consists of an inlet tube, a reactor chamber containing the wafer and an outlet tube. 


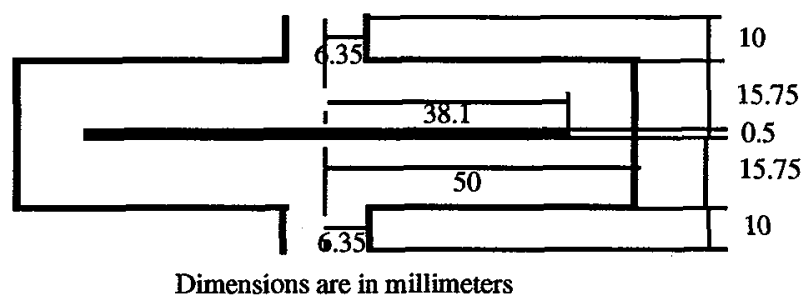

Fig. 1 Rapid thermal CVD reactor

\section{3.- Chemical mechanism.}

During CVD process, two types of chemical reactions take place : gas phase reactions and solid surface ones.

\section{Gas phase reactions :}

In the case of deposition of silicon from silane $\left(\mathrm{SiH}_{4}\right)$, it was found that higher order silane can be present in the gas phase. However, most studies agree on the fact that only silane, disilane $\left(\mathrm{Si}_{2} \mathrm{H}_{6}\right)$, silylene $\left(\mathrm{SiH}_{2}\right)$ and hydrogen $\left(\mathrm{H}_{2}\right)$ cannot be neglected in a representation of the gaseous phase. That is why only the two following chemical reactions are considered :

$$
\begin{aligned}
& \mathrm{SiH}_{4} \leftrightarrow \mathrm{SiH}_{2}+\mathrm{H}_{2} \\
& \mathrm{Si}_{2} \mathrm{H}_{6} \leftrightarrow \mathrm{SiH}_{2}+\mathrm{SiH}_{4}
\end{aligned}
$$

The forward and reverse reaction rate constants, as calculated by Fayolle [2] using quantum RRK (Rice-Ramsperger-Kassel) method, are function of temperature and total pressure.

\section{Solid surfaces reactions :}

On solid surfaces, the contributions to the silicon deposition of all the species produced in the gas phase are taken into account, according to the following heterogeneous chemical reactions :

$$
\begin{aligned}
& \mathrm{SiH}_{4} \rightarrow \mathrm{Si}+2 \mathrm{H}_{2} \\
& \mathrm{SiH}_{2} \rightarrow \mathrm{Si}+\mathrm{H}_{2} \\
& \mathrm{Si}_{2} \mathrm{H}_{6} \rightarrow 2 \mathrm{Si}+3 \mathrm{H}_{2}
\end{aligned}
$$

The growth rate of silicon from silane, reaction $\mathrm{R} 3$, has been calculated using the kinetic equation established by Wilke et al. [3].

Silylene is known to be a very reactive radical. Its deposition rate corresponding to reaction $R 4$ is supposed to be first order and is calculated using a sticking coefficient equal to unity [4].

For disilane, it is well known that this product constitutes a more reactive chemical source than silane for silicon deposition on surfaces [5]. But it appears that these higher deposition rates result not from disilane itself but from its homogeneous pyrolysis products [6]. For this work, the hypothesis has been made that disilane itself is not reactive on surfaces and its direct influence by reaction $R 5$ has been neglected. 


\section{4.- Mathematical model.}

The two models (specific program and software package) used to simulate the fluid flow, heat and mass transport phenomena in the rapid thermal CVD reactor presented on Fig. 1, consist of a set of partial differential equations associated to appropriate boundary conditions and satisfying several assumptions : stationnary regime, laminar flow, no heat transfer of the gas by radiation, heats of reactions and variations of physical properties with concentrations of the reactive species are neglected. These equations can be written as :

$$
\begin{aligned}
& \nabla \rho \vec{v}=0 \\
& \rho \frac{D \vec{v}}{D t}=-\nabla p-[\nabla \cdot \vec{\tau}]+\rho \vec{g} \\
& \rho c_{p} \frac{D T}{D t}-k \nabla T=0 \\
& \nabla\left(c y_{k} \vec{v}\right)-\nabla\left(c D_{k m} \nabla y_{k}\right)-R_{v k}=0
\end{aligned}
$$

\section{Boundary conditions :}

At the reactor and substrate walls, we assume a no-slip conditions for the velocity components, a fixed temperature based on experimental data and heterogeneous reactions for each species with an infinite rate for silicon deposition from silylene.

At the reactor inlet, the velocity, temperature and flow rate are fixed.

At the reactor outlet, zero axial gradients of velocity, temperature and concentration are used.

At the centerline, no radial gradients of velocity, temperature and concentration are considered.

These boundary conditions are those adopted by the specific program. The same conditions are considered in the software package except on solid surfaces where a finite rate for silicon deposition from silylene is taken into account and at the reactor outlet where only pressure is specified.

\section{Specific program :}

The specific program developed by Magnaudeix [1] is especially applicable for axisymmetrical cold wall chemical vapor deposition (CWCVD) reactors. In this code, the discretization of the mathematical equations (eq. 1 to eq. 4) described above, has been realized by a control volum method.

The momentum equation (eq. 1 and eq. 2) and the energy one (eq. 3) have been solved by the so-called simpler algorithm of Patankar and Spalding [7]. Then the concentration profiles of each chemical species are derived from the resolution of mass transfer equations (eq. 4) through a line-by-line method.

\section{Software package :}

Many powerful codes have been developed and are commercially available : FIDAP (USA), PHOENICS (GB) and FLUX EXPERT (FRANCE). In this work another software called ESTET, elaborated in FRANCE by EDF and commercialized by SIMULOG is used. It permits a three dimensional treatment of fluid mechanics problems coupled to heat transfer.

In ESTET, equations are discretized using a combination of finite differences and finite volumes methods. The resolution is based on the fractionnary step method (Chorin method) which allows to select appropriate solvers for each term of each equation. 
The velocity and temperature profiles as previously determined, are then used to solve the mass transfer equations written for each chemical species. The equations are discretized using finite differences. In our case, the use of centered differences to approximate the convection term causes divergence. As a remedy to these numerical problems we have used an upwind scheme in which the forward and backward differences approximations are applied to the convection term while standard centered differences are applied to the other terms. Finally, the resulting algebraic equations have been solved iteratively by a line-by-line under-relaxation procedure.

\section{5.- Results and discussion.}

A very large number of informations can be deduced from the analysis of the modeling results. Before presenting some of them, we will describe one particular set of results which will constitute a basis for comparison in this study. This simulation corresponds to the following operating conditions :

total pressure $: p=0.1$ Torr, substrate temperature : $T_{s}=1073 \mathrm{~K}$, reactor wall temperature $T_{w}=373 \mathrm{~K}$, inlet flow rate $: q=200 \mathrm{sccm}$.

Figure 2 presents the streamline profiles. We can observe that the gas follows smoothly solid surfaces in a creeping regime in all parts of the reactor except in the region situated just after the inlet tube where a recirculating zone is detected. This zone tends to disappear when the inlet flow rate decreases to $100 \mathrm{sccm}$. Because of the very low pressure in the reactor, Reynolds number never exceeds the value of 18 and the flow regime remains laminar in all parts of the reactor.

The temperature distribution in the reactor is illustrated in Fig. 3. The first remark that can be made is the asymmetry of the isotherms. In the entrance region, the cool gas arrives by forced convection and becomes progressively heated as it approaches the wafer, causing then a reduction of the distance between the isotherms. Below the wafer the effect is inverted. In fact, we can observe that the gas temperature decreases during the flow to the exhaust and the isotherms are more spaced than those situated above the substrate.

For chemical species we only present the concentration profiles of silylene (Fig. 4). The evolutions of the silane concentration are not illustrated here by figures, but need a few comments. In all cases studied here, the silane concentration remains at a high value even at the reactor end. Its very low consumption is essentially due to surface reactions, giving the main contribution to silicon deposition. The influence of the operating conditions observed on the concentration profiles are only kinetical influences on the growth rate and will not be discussed in this paper. But for silylene, a very reactive radical contributing to silicon deposition, the behavior is different and more complex. Its profile depends closely on the distribution of the temperature in the reactor. Above the wafer the maximum of silylene concentration is situated in the middle between the axis and the edge of the wafer. This is respectively due to the low value of the temperature caused by the incoming cool gas near the axis and to the cold wall of the reactor. Below the wafer, the highest value of silylene concentration is located near the axis, as can be predicted because of the thermal effect.

The contribution of silylene to silicon deposition is presented on Fig. 5 for the two types of surface conditions used in the models. It is important to emphazise that considering an infinite rate for silylene decomposition on the surface gives a growth rate of silicon ten times greater than the growth rate calculated using a finite rate based on the kinetic theory of the gas. In both cases, the silylene contribution remains very low compared to silane contribution and can be neglected when silicon deposition from silane is studied. 
But silylene plays an important role in process deposition of silicon where silane is inhibited such as for phosphorus in situ doped silicon deposition.

\section{Pressure and substrate temperature effects :}

The value of total pressure in the reactor has a great influence on chemical species concentrations. By increasing total pressure the gas residence time in the reactor becomes longer and the reactivity on surfaces takes a greater importance.

Concerning the thermal effect, it is important to specify that because the temperature of the reactor wall has been kept constant at $373 \mathrm{~K}$ and due to the great difference between the area of the substrate $\left(9.1210^{-3} \mathrm{~m}^{2}\right)$ and the area of the reactor wall $\left(2.6310^{-2} \mathrm{~m}^{2}\right)$, the hot region where chemical reactions occur in the reactor is limited to a few millimeters around the substrate.

Figure 6 presents the distribution of silylene concentration for the following operating conditions : inlet flow rate : $100 \mathrm{sccm}$, substrate temperature : $1073 \mathrm{~K}$, total pressure : 0.5 Torr. Compared to the profile given on Fig. 4, we can observe, first, that an increase of pressure in the reactor from 0.1 Torr to 0.5 Torr produces a contraction of the isoconcentrations on the wafer and, second, that a decrease of the inlet total flow rate from $200 \mathrm{sccm}$ to $100 \mathrm{sccm}$ causes a displacement of the maximum of silylene concentration above the wafer. This can be explained by the increase of the residence time so that the gas is heated sufficiently in the entrance zone. The same effect can be observed on Fig. 7 corresponding to an inlet flow of $100 \mathrm{sccm}$, a substrate temperature of $1073 \mathrm{~K}$ and a total pressure of 0.1 Torr.

For chemical species, an increase of total pressure or an increase of substrate temperature produces a consumption of silane then a production of silylene. The table below shows some important values of silane and silylene molar fraction.

\begin{tabular}{|c|c|c|}
\hline & $\begin{array}{c}\text { Minimum of silane } \\
\text { molar fraction }\end{array}$ & $\begin{array}{c}\text { Maximum of silylene } \\
\text { molar fraction }\end{array}$ \\
\hline $\begin{array}{c}\text { Total pressure } \\
\text { (Torr) }\end{array}$ & \multicolumn{2}{|c|}{$\begin{array}{c}\text { Inlet flow rate }: 100 \mathrm{sccm} \\
\text { Substrate temperature }: 1073 \mathrm{~K}\end{array}$} \\
\hline 0.1 & 0.993 & $1.2110^{-7}$ \\
\hline 0.2 & 0.990 & $3.4310^{-7}$ \\
\hline 0.3 & 0.985 & $6.1510^{-7}$ \\
\hline 0.4 & 0.981 & $9.3610^{-7}$ \\
\hline 0.5 & 0.976 & $1.1910^{-6}$ \\
\hline substrate temperature & Inlet flow rate : $100 \mathrm{sccm}$ \\
\hline$(\mathrm{K})$ & Total pressure : 0.1 Torr \\
\hline 873 & 0.999 & $1.1610^{-9}$ \\
\hline 1073 & 0.993 & $1.2110^{-7}$ \\
\hline 1173 & 0.989 & $6.7810^{-7}$ \\
\hline 1273 & 0.982 & $2.9010^{-6}$ \\
\hline 1373 & 0.975 & $1.0010^{-5}$ \\
\hline
\end{tabular}

The main information given in this table is that an increase of pressure or of substrate temperature, produces an increase in silane consumption. That is the reason that the silicon deposition due to this species increases with these two physical parameters. At the same time, the consumption of silane remains slight (it does not exceed 3\%) so the deposition is however uniform. On the other hand, the concentration of silylene varies 
highly and the resulting silicon deposition is not uniform but its contribution to the total deposition rate in the working conditions remains smaller than 1\% (Fig. 8 and Fig. 9).

\section{6.- Conclusion.}

An important mathematical tool has been developed in this work permitting us to get two calculation codes. Both of them give the same results for velocity and temperature distribution in the reactor while a difference in chemical species concentrations can be observed. This disagreement does not implicate the accuracy of one of the codes, but it is essentially due to the boundary conditions used for silylene on solid surfaces. CWCVD considers a total consumption of silylene produced in the gas phase on the solid surfaces and ESTET chooses a finite rate calculated on the basis of the kinetic theory of gases using a sticking coefficient value. The main advantage of ESTET is that it can treat a great variety of three dimensional phenomena occuring in different equipments, but it necessitates a long calculation time. Whereas, CWCVD treats only some specific axi-symmetrical CVD reactors and then its execution time is short.

The main results that can be emphazised in this work follows the fact that the RTCVD reactor studied constitutes a type of cold wall reactors. In this equipment, the gas mixture remains cool except in the regions situated immediatly around the substrate so that the homogeneous reactions produce a small quantity of silylene and other radicals. This phenomenon puts in evidence the interests of RTCVD reactors which are particularly adapted for the deposition from thermosensible gases such as organometallics. When the chemical mechanism of a particular system is known, the models presented can predict the growth rate distribution and then they can optimize it in function with operating conditions.

\section{List of symbols}

$\begin{array}{ll}c & \text { molar concentration } \\ c_{p} & \text { gas mixture specific heat } \\ \mathbf{D}_{\mathbf{k}} & \text { binary diffusion coefficient of species } \mathbf{k} \text { to gas mixture } \\ \overrightarrow{\mathrm{g}} & \text { gravitational acceleration } \\ \mathbf{k} & \text { gas mixture thermal conductivity } \\ \mathbf{p} & \text { total pressure } \\ \mathbf{R}_{\mathrm{vk}} & \text { molar rate of production of species } \mathbf{k} \\ \mathbf{T} & \text { gas mixture temperature } \\ \mathbf{t} & \text { time } \\ \overrightarrow{\mathrm{v}} & \text { vector velocity } \\ \mathrm{yk} & \text { molar fraction of species } \mathbf{k} \\ \overrightarrow{\vec{\tau}} & \text { stress tensor } \\ \rho & \text { gas mixture density }\end{array}$

\section{References}

[1] Magnaudeix S., Doctorat thesis, ENSIGC, France (1992).

[2] Fayolle F., Doctorat thesis, ENSIGC, France (1993).

[3] Wilke T.E., Turner R.A., and Takoudis C.J., Chem. Eng. Sci., 144 (1986) 643.

[4] Meyerson B.S., Scott B., and Tsui R., Chemtronics, 1 (1986) 150.

[5] Coltrin M.E., Kee R.J., and Miller A., J. Electrochem. Soc., 133 (1986) 1206.

[6] Gueye M., Scheid E., Taurines P., Duverneuil P., Bielle-Dapet D., and Couderc J.P.,J. Phys., 1 (1991) 63.

[7] Patankar S.V., and Spalding D.B., Numerical Heat Transfer and Fluid Flow, McGraw-Hill, New York (1980). 


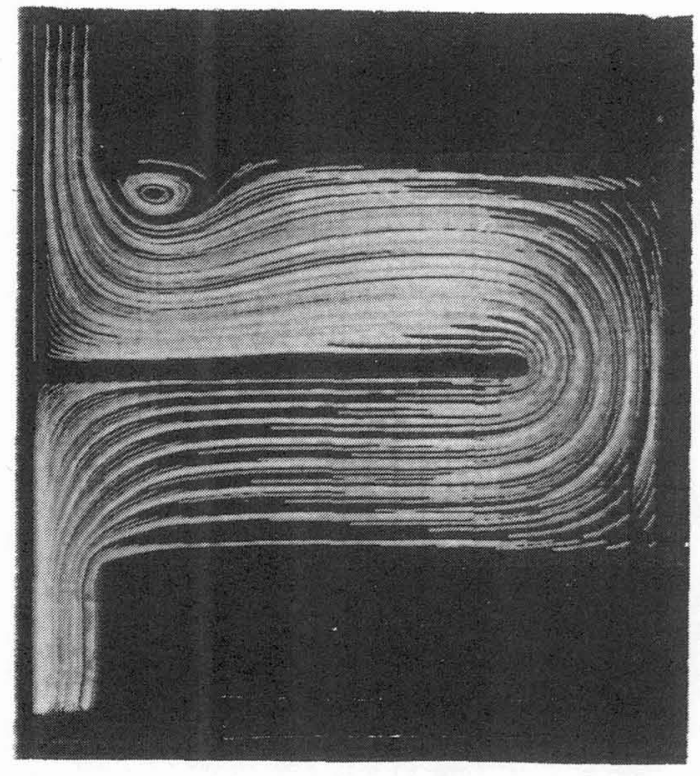

Fig. 2. Streamlines in the RTCVD reactor for $\mathrm{p}=0.1$ Torr, $\mathrm{Ts}=1073 \mathrm{~K}, \mathrm{q}=200 \mathrm{sccm}$.

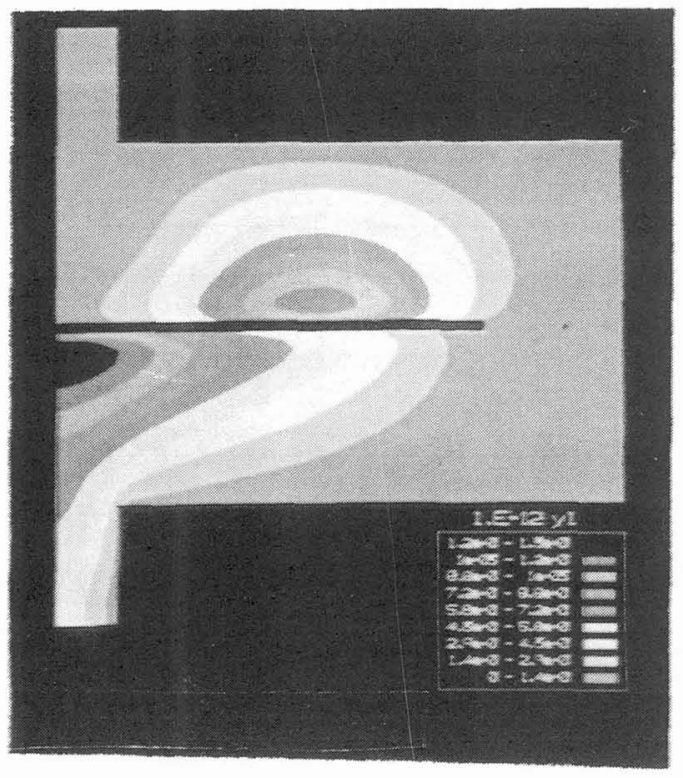

Fig. 4. Silylene molar fraction in the RTCVD reactor for $p=0.1$ Torr, $T s=1073 \mathrm{~K}, q=200 \mathrm{sccm}$.

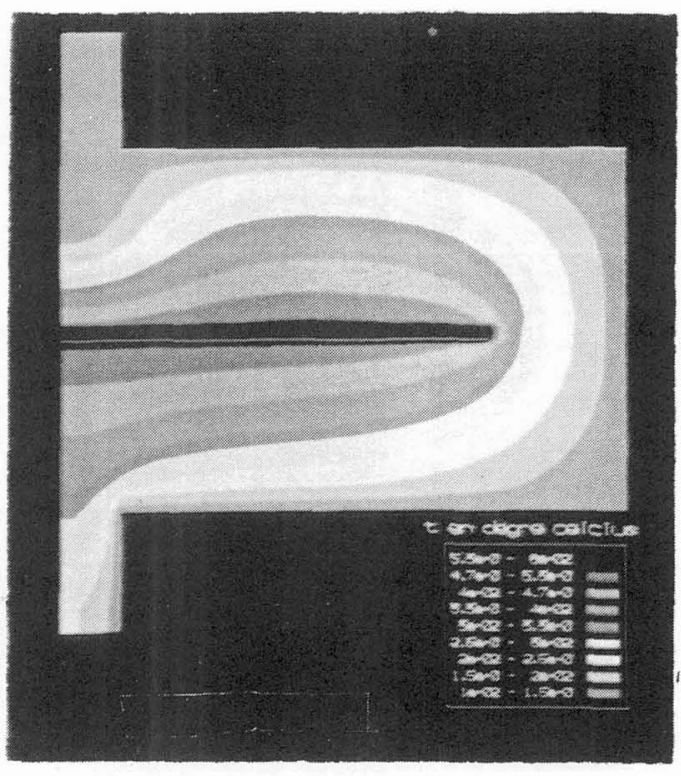

Fig. 3. Thermal map in the RTCVD reactor for $p=0.1$ Torr, $T s=1073 \mathrm{~K}, q=200 \mathrm{sccm}$.

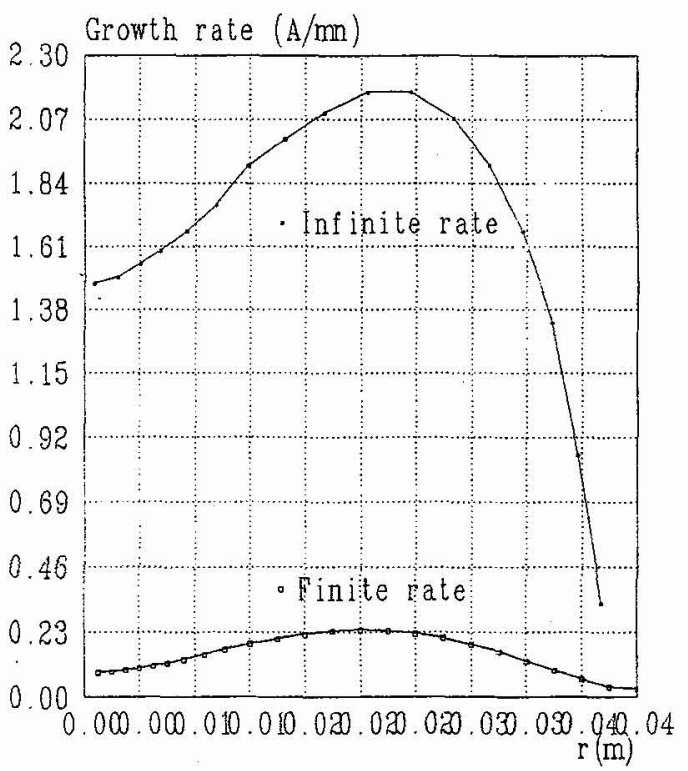

Fig. 5. Silicon growth rate from silylene in the RTCVD reactor at an infinite and a finite rate. 


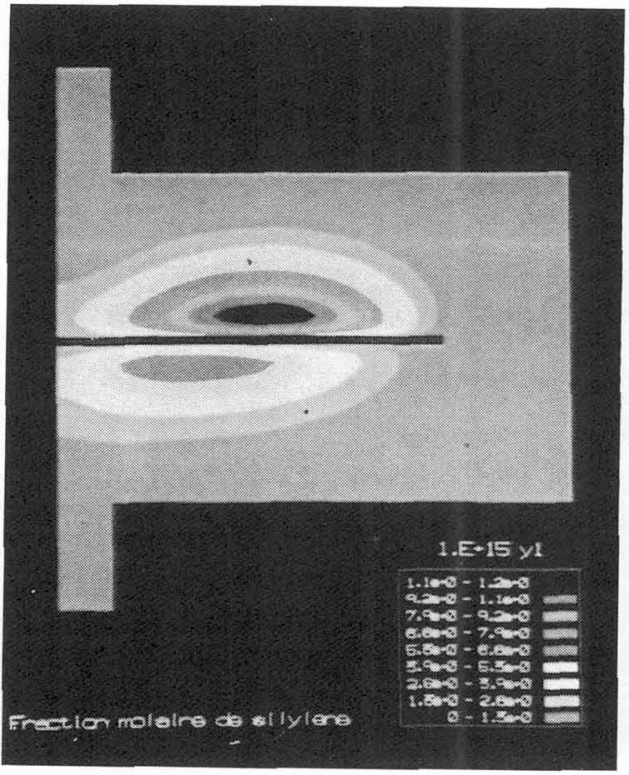

Fig. 6. Silylene molar fraction in the RTCVD reactor for $\mathrm{p}=0.5$ Torr, $\mathrm{Ts}=1073 \mathrm{~K}, \mathrm{q}=100 \mathrm{sccm}$.

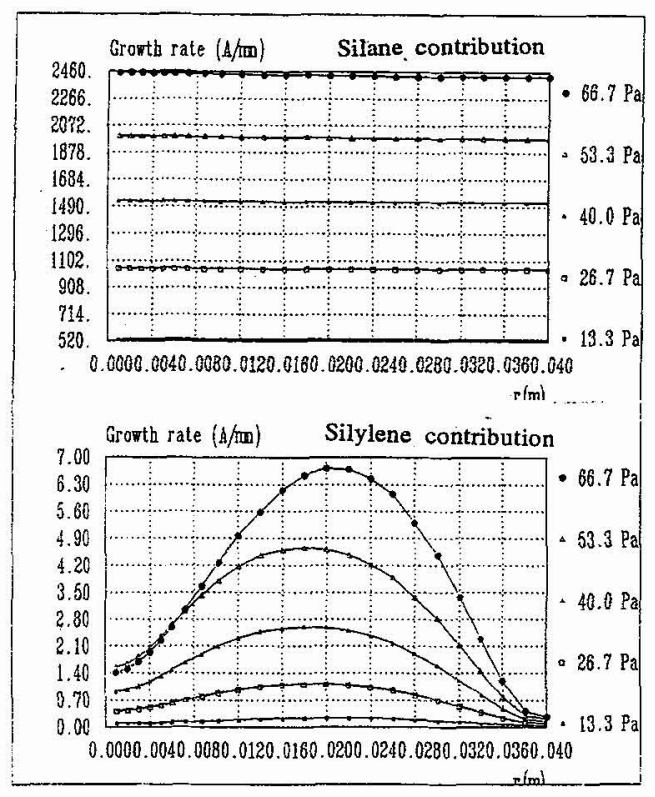

Fig. 8. Total pressure effect on silicon growth rate in the RTCVD reactor.

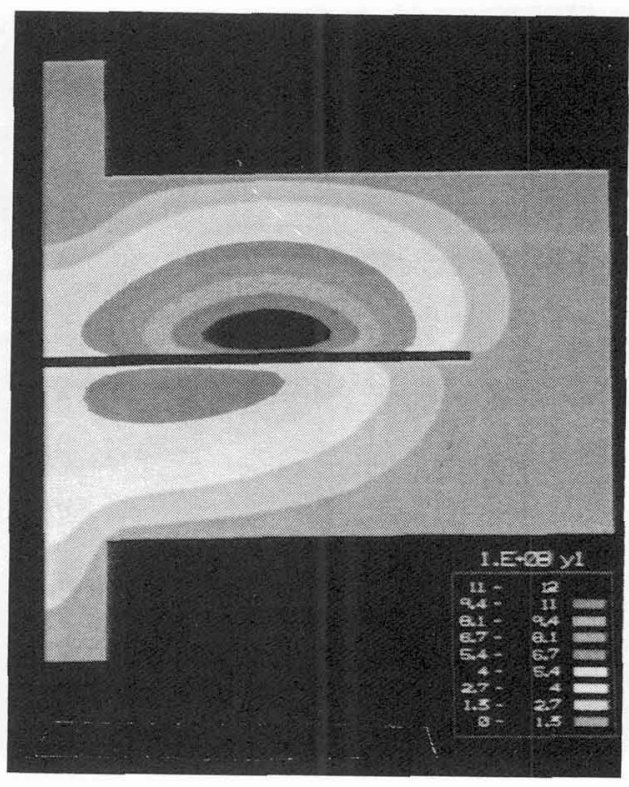

Fig. 7. Silylene molar fraction in the RTCVD reactor for $p=0.1$ Torr, $T s=1073 \mathrm{~K}, q=100 \mathrm{sccm}$.

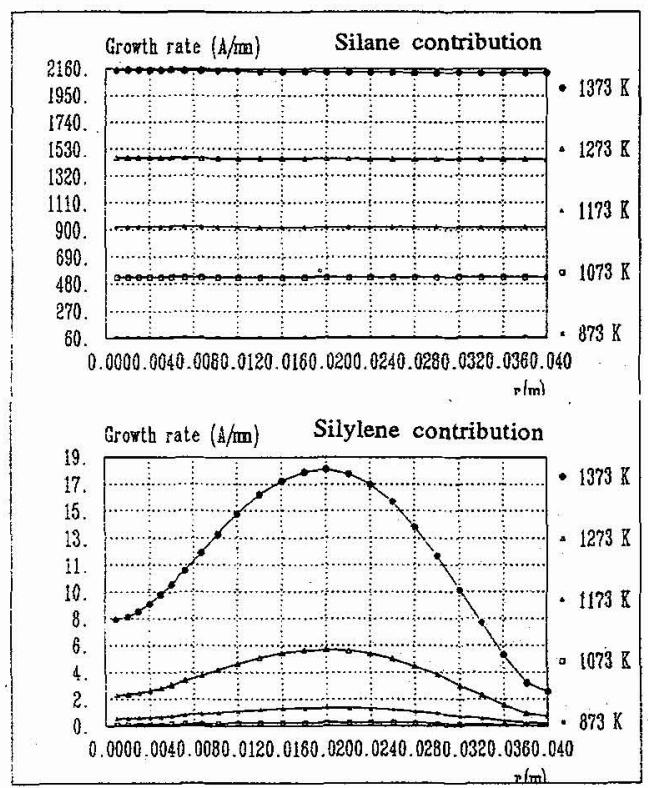

Fig. 9. Susceptor temperature effect on silicon growth rate in the RTCVD reactor. 